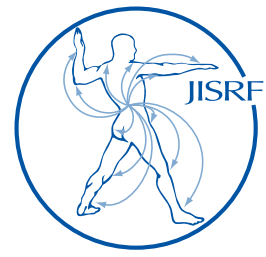

\title{
Diagnosing and Treating Popliteal Tendinopathy After Total Knee Arthroplasty
}

Martin J ${ }^{1}$, Fout $A^{2}$, Stoeckl $A^{2}$, Dennis $D^{1}$

\begin{abstract}
The following office tip describes four patients that underwent primary total knee arthroplasty and developed posterolateral knee pain at a mean follow-up duration of 1.6 months postoperatively. The first patient in this series noted substantial pain lying in bed (in a lateral decubitus position with the operative leg up) while attempting to abduct her leg to adjust her sheet in bed. A thorough clinical and radiographic work-up was performed. This patient's posturing in bed (and subsequent physical exam maneuver) led to a presumptive diagnosis of popliteal tendinopathy. The diagnosis was confirmed arthroscopically by identifying a frayed and inflamed popliteal tendon. After undergoing arthroscopic popliteal tendon release, the patient noted complete pain relief while retaining coronal stability in both flexion and extension. The following office tip defines a previously undescribed clinical diagnostic examination for popliteal tendinopathy that was identified based on a patient's symptomatology and subsequently utilized to identify three additional cases of arthroscopically confirmed popliteal tendinopathy.
\end{abstract}

Keywords: total knee arthroplasty; popliteal tendinosis; popliteal tendonitis; tendinopathy; posterolateral knee pain Level of Evidence: AAOS Therapeutic Level IV

\section{Introduction}

Pain following primary total knee arthroplasty (TKA) is unfortunately a common finding [1]. There are numerous causes for a painful TKA, which make diagnosing the source of the pain even more challenging $[\underline{2}, \underline{3}]$. Popliteal tendinopathy is an uncommon cause of pain, which can present with posterolateral knee pain, often associated with motion-induced crepitus in the region of the popliteal tendon. The first description of popliteal tendon dysfunction during primary TKA was noted intraoperatively at the time of capsular closure [4]. The authors noted a "popping" that occurred during flexion and extension. After release of the popliteal tendon, they noted that the "popping" disappeared and that the knee was stable. Since this study, Insall et. al.
1 J. Ryan Martin, MD; Douglas A Dennis, MD Colorado Joint Replacement, Denver, CO 80210

2 Allison Fout, PA-C; Andrew C Stoeckl, MD Excelsior Orthopaedics, Amherst, NY 14226

(Direct reprint requests to J. Ryan Martin)

(C) 2017 Martin, Fout, Stoeckl, Dennis. All rights reserved. Authors retain copyright and grant the journal right of first publication with the work. Reconstructive Review is an open access publication and follows the Creative Commons Attribution-NonCommercial CC BY-NC. This license allows anyone to download works, build upon the material, and share them with others for noncommercial purposes as long as they credit the senior author, Reconstructive Review, and the Joint Implant Surgery \& Research Foundation (JISRF). An example credit would be: "Courtesy of (senior author's name), Reconstructive Review, JISRF, Chagrin Falls, Ohio". 
proposed arthroscopic popliteal tendon release for patients diagnosed with popliteal tendon dysfunction following primary TKA [5]. However, to our knowledge, other than the presence of patient-reported posterolateral discomfort, we are unaware of any single clinical examination maneuver to precisely diagnose popliteal tendinopathy following primary TKA. The following office tip describes a clinical examination maneuver utilized to diagnose four patients with popliteal tendinopathy, and secondarily confirmed at the time of arthroscopic release of the popliteal tendon.

\section{Office Tip}

The diagnosis of post-operative popliteal tendinopathy following total knee arthroplasty can be made with a simple clinical test. Each case of popliteal tendinopathy was confirmed arthroscopically and after release of the tendon, resulted in complete relief of symptoms.

\section{Case Examples}

All but one of the patients in this series underwent pri- mary TKA by one of the senior authors at a single institution. The primary TKA was performed utilizing a gap balancing approach with a posterior stabilized mobile bearing Attune TKA (Warsaw, IN). The postoperative course was uneventful in each case. However, approximately 2 months postoperatively, the first patient in this series presented with posterolateral knee pain. This patient was a 70 year old female with a preoperative diagnosis of osteoarthritis of her right knee. Preoperative radiographs of her right knee demonstrated tricompartmental arthritis and chondrocalcinosis (Figure 1). Postoperative radiographs of her right knee demonstrated a well-fixed, well-aligned TKA without evidence of loosening, lysis, lateral overhanging femoral component, or lateral femoral osteophytes (Figure 2).

During her clinical evaluation, she localized the pain to the posterolateral aspect of her knee, and noted no significant pain when ambulating or sitting. Her pain could not be reproduced on clinical examination, although the posterolateral aspect was diffusely painful to palpation. Interestingly, she noted substantial pain lying in a lateral decubitus position in bed with the operative extremity up when abducting her leg while attempting to adjust the bed sheets (Figure 3). Repeat of this maneuver consistently repro-

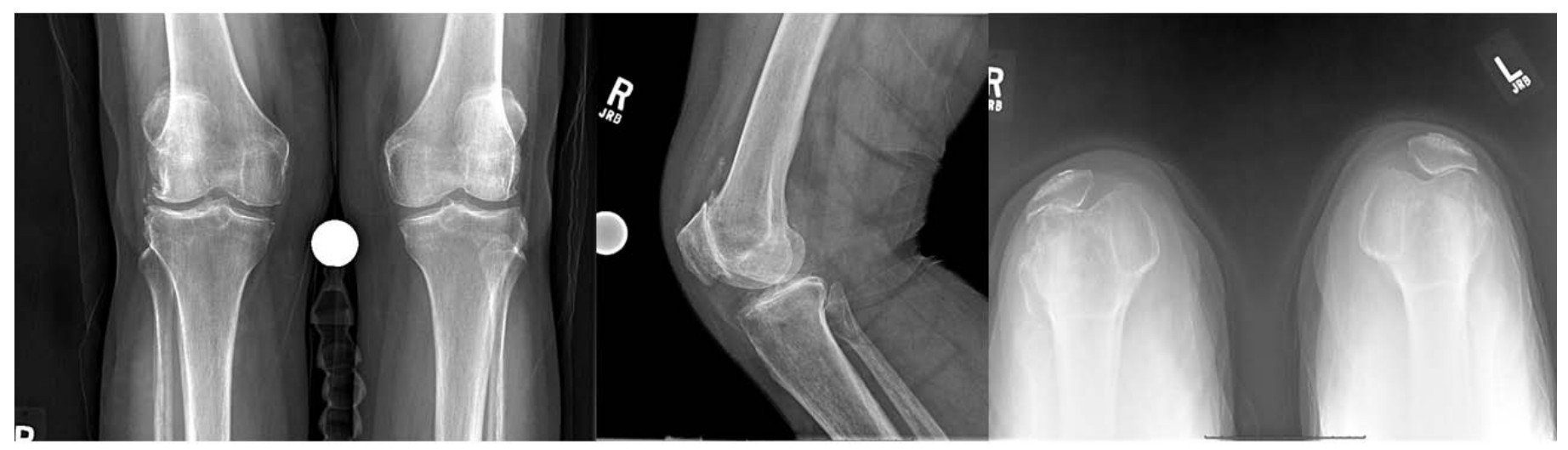

Figure 1. AP, lateral and patellar view of the right knee demonstrates severe patellofemoral arthritis with chondrocalcinosis.

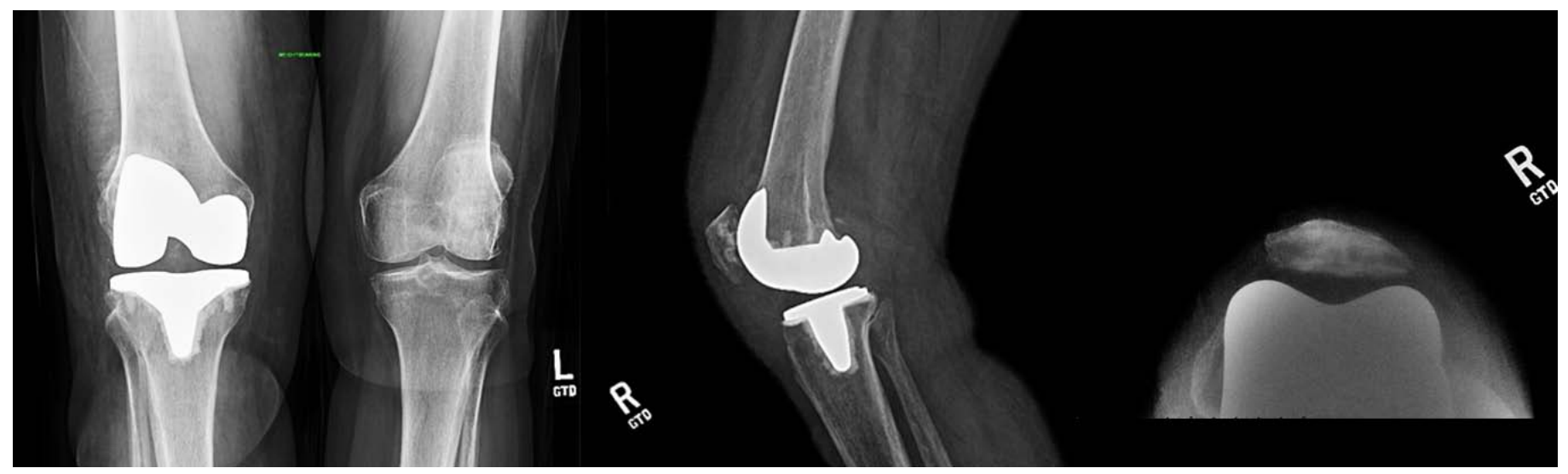

Figure 2. Postoperative AP and patellar view demonstrate a well-fixed well-aligned right total knee arthroplasty. There does not appear to be evidence of lateral femoral osteophytes or femoral component overhang. 


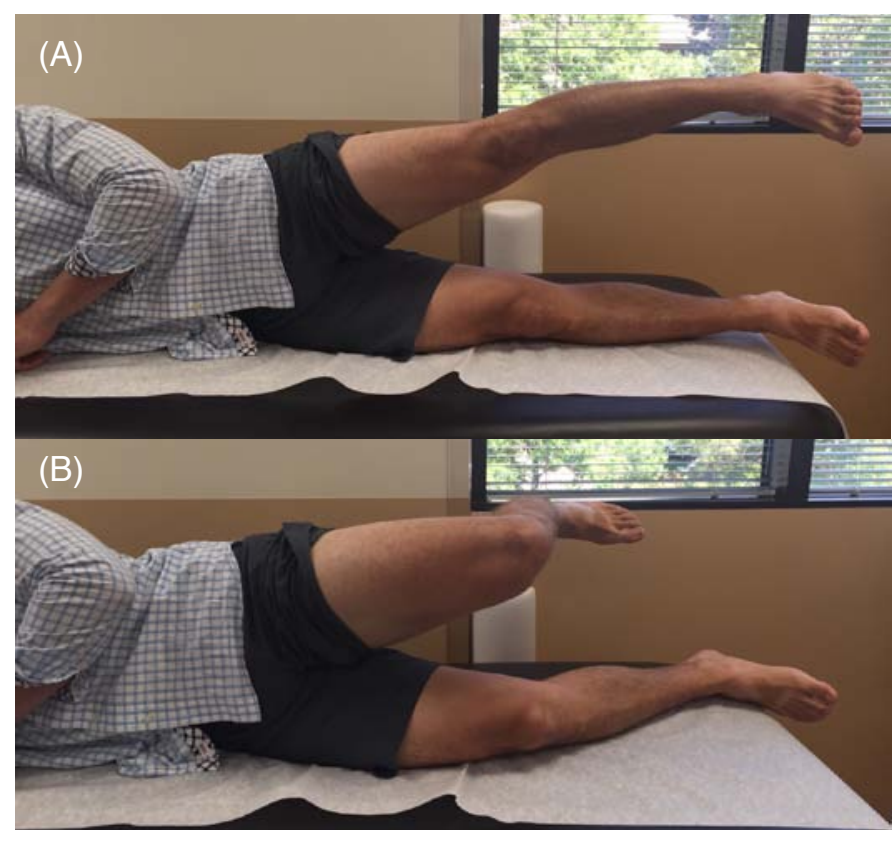

Figure 3. The above clinical photographs demonstrate the clinical exam maneuver for diagnosing popliteal tendonitis. (A) The patient is placed in a lateral decubitus position with the operative extremity up. The patient then abducts their hip against gravity resistance with their knee in full extension. (B) With their hip abducted the patient flexion their knee from 0 to 90 degrees.

duced the posterolateral pain. This position applied a varus stress to a flexed knee, a position where the popliteal tendon acts an important static restraint in varus stability []․ Therefore, it was hypothesized that this maneuver could be utilized to assess for popliteal tendinopathy in the setting of TKA. The patient was treated with conservative measures including activity modification, icing, and NSAID use for three months with continued pain.

After discussing the proposed mechanism for posterolateral pain to this patient, an arthroscopic release of the popliteal tendon was recommended and performed seven months after primary TKA. Intraoperatively, the popliteal tendon was visualized and noted to have substantial tendonitis, fraying, and was shown to impinge slightly on the femoral component of the TKA (Figure 4). Utilizing the arthrosopic shaver, the popliteal tendon was released without any additional procedures performed.

After the arthroscopic popliteal tendon release, the patient noted complete relief of her posterolateral pain within 48 hours following the operative procedure. On her most recent clinical appointment, six months after surgery, she was noted to have complete resolution of her pain. Additionally, her knee was stable to varus and valgus stresses in flexion and extension. Her radiographs demonstrated no change, with continued excellent alignment and no evidence of radiolucencies or lysis.

Since the first patient presentation, three additional pa-

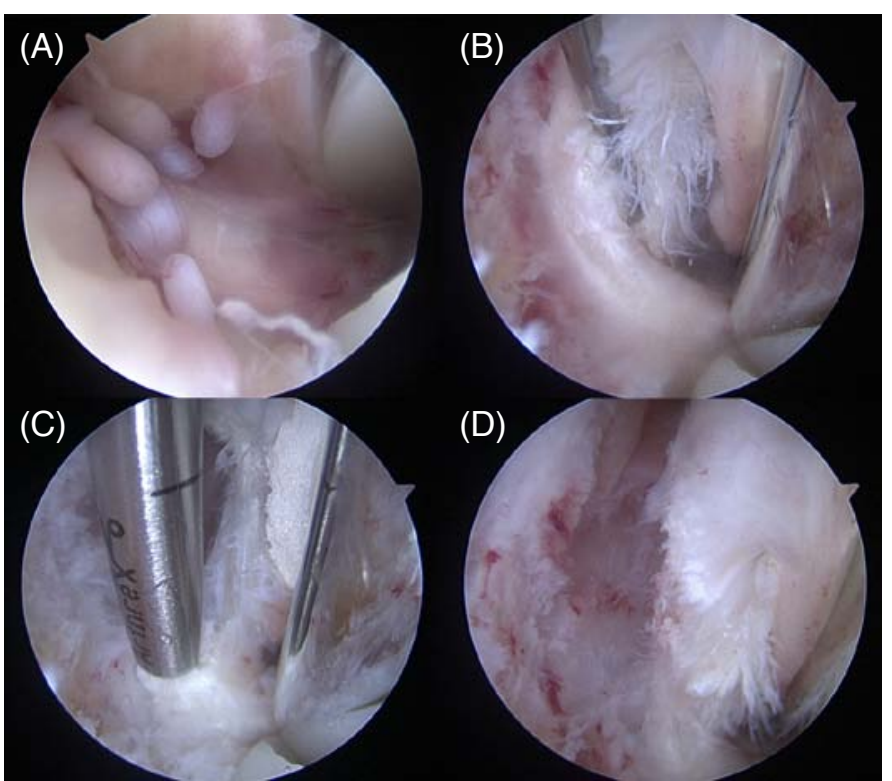

Figure 4. Arhtroscopic views of the right knee. A: The lateral compartment of the right demonstrates substantial synovitis and $(B)$ fraying of the popliteal tendon. C: The arthroscopic shaver is utilized to resect the popliteal tendon. D: The popliteal tendon has been completely released.

tients presented with similar posterolateral knee pain. Utilizing the clinical examination maneuver described above with a varus stress applied to the patient's operative extremity in a lateral decubitus position, each patient's pain was readily reproduced. The average duration from TKA to the diagnosis of popliteal tendonitis was 1.6 months. The arthroscopic findings in each patient were diagnostic of popliteal tendinopathy. Subsequently, all patients noted complete or near complete resolution of their pain without any evidence of coronal or sagittal instability identified at final follow-up.

\section{Discussion}

Postoperative knee pain following primary TKA is unfortunately a common finding [1]. Furthermore, identifying the source of pain can occasionally be a diagnostic dilemma due to the wide variety of pathologies that can lead to knee pain in this patient population $[\underline{2}, \underline{3}, \underline{7}]$. One uncommon source of postoperative knee pain is popliteal tendinopathy []. Although there have been case reports describing treatments for popliteal tendon dysfunction, little is known about how to best identify this clinical condition precisely [4]. To our knowledge, a diagnostic clinical examination maneuver for popliteal tendinopathy following TKA has yet to be described prior to this study. Therefore, the following case series describes a unique clinical technique of identifying popliteal tendon dysfunction with sub- 
sequent arthroscopic confirmation of the diagnosis. Additionally, a short-term clinical and radiographic follow-up was performed on this patient population to ensure resolution of symptoms.

The proposed diagnostic maneuver requires the patient to lie supine with their operative extremity elevated. The examiner then has the patient abduct the operative extremity while an adduction force is applied to the lower leg close to the ankle joint. This force results in a varus stress through the knee. As was discussed previously, the popliteal tendon is an important varus stabilizer in knee flexion [] $]$. Therefore, this clinical test appears to specifically stress the popliteal tendon. Each patient in this series had nonspecific posterolateral knee pain, which could not be reliably reproduced with common clinical examination maneuvers that apply varus and valgus stresses in flexion and extension. After discovering this maneuver during the first patient encounter in this series, all subsequent patients stated that their pain was reliably reproduced.

The source for popliteal tendinopathy has been attributed to a retained lateral femoral condylar osteophyte or posterior femoral component impingement, often associated with excessive lateral positioning of the femoral component beyond the osseous perimeter of the lateral femoral condyle [ [ ] $]$. Both of these conditions can increase tension on the traversing popliteal tendon resulting in irritation. Additionally, arthroscopic release of the popliteal tendon was proposed as a standard treatment for patients diagnosed with popliteal tendinopathy [ $\underline{8}]$. The release of the tendon in this patient population was not associated with an increase in instability and had reliable pain relief. However, a recent study demonstrated a decrease in patient outcomes at two to three years postoperatively in a cohort of 15 patients that had accidental iatrogenic intraoperative popliteal tendon release [9]. Although assessing a different outcome, these results appear to differ from Kesman et. al., who prospectively enrolled eighteen patients to randomly have their popliteal tendon released by the first assistant, while the senior surgeon was blinded to the presence or absence of the release [10]. The senior surgeon, with trials in place, would assess ligamentous stability. No subjective difference in static stability was noted between the two cohorts. It is possible that the arthroscopic release and the intraoperative isolated release of the popliteus tendon are more precise methods for releasing the popliteal tendon. Whereas the iatrogenic, saw blade release, of the popliteal tendon could potentially release the posterolateral corner of the knee resulting in worse outcomes. However, longer outcomes are necessary to determine if an arthroscopic release of the popliteal tendon will impact patient outcomes.

There are several notable limitations to the following study. First, this was a retrospective case series, and therefore has inherent limitations of both the study design and the small patient population. However, we still believe this diagnostic maneuver is useful in identifying popliteal tendinopathy, an uncommon cause of postoperative knee pain. Second, this clinical test has not been validated or compared to other diagnostic tests. Therefore the diagnostic accuracy is unknown. Although we did note arthroscopic evidence of popliteal tendinopathy and complete pain relief after release in each patient, a comparative study including imaging and other exam maneuvers to determine the validity of this exam are warranted. Third, it is possible that each patient's tendinopathy may have resolved without surgical treatment. Future studies should be centered on the natural progression of popliteal tendinopathy after total knee arthroplasty which may help direct the timing of surgical intervention in this patient population. Finally, long-term clinical follow-up is necessary to determine if there are any clinical consequences of popliteal tendon release. As was discussed previously, one study noted worse outcomes in patients that had iatrogenic popliteal tendon release during primary TKA [9]. This may represent a posterolateral corner release and therefore may not be generalizable to the isolated popliteal tendon release noted in this study.

\section{Summary}

The following study demonstrates a clinical examination method for diagnosing popliteal tendon dysfunction. This exam was performed on four patients in this series and the diagnosis of popliteal tendinopathy was confirmed arthroscopically in each case. An arthroscopic popliteal tendon release was subsequently performed and resulted in complete pain relief in all patients. Additionally, no postoperative instability was identified. This clinical examination should be considered and utilized when confronting the common diagnostic dilemma of a painful TKA, especially posterolateral knee pain that is difficult to reproduce clinically.

\section{Disclosure}

The authors declare that there is no conflict of interest regarding the publication of this paper. For full disclosures refer to last page of this journal. 


\section{References:}

1. Gandhi R, Davey JR, Mahomed NN. Predicting patient dissatisfaction following joint replacement surgery. J Rheumatol. 2008;35(12):2415-2418. doi:10.3899/ jrheum.080295.

2. Dennis DA. Evaluation of painful total knee arthroplasty. J Arthroplasty. 2004;19(4 Suppl 1):35-40. http://www.ncbi.nlm.nih.gov/pubmed/15190547. Accessed June 17, 2016.

3. Potty AGR, Tzeng TH, Sams JD, et al. Diagnosis and Management of Intra-articular Causes of Pain After Total Knee Arthroplasty. Instr Course Lect. 2015;64:389401. http://www.ncbi.nlm.nih.gov/pubmed/25745923. Accessed June 17, 2016.

4. Barnes CL, Scott RD. Popliteus tendon dysfunction following total knee arthroplasty. J Arthroplasty. 1995;10(4):543-545. http://www.ncbi.nlm.nih.gov/ pubmed/8523017. Accessed May 2, 2016.

5. Allardyce TJ, Scuderi GR, Insall JN. Arthroscopic treatment of popliteus tendon dysfunction following total knee arthroplasty. J Arthroplasty. 1997;12(3):353-355. http://www.ncbi.nlm.nih.gov/pubmed/9113555. Accessed June 17, 2016.
6. Nielsen S, Helmig P. The static stabilizing function of the popliteal tendon in the knee. An experimental study. Arch Orthop Trauma surgery Arch für orthopädische und Unfall-Chirurgie. 1986;104(6):357-362. http://www.ncbi.nlm.nih.gov/ pubmed/3964042. Accessed June 17, 2016.

7. Manning BT, Lewis N, Tzeng TH, et al. Diagnosis and Management of Extra-articular Causes of Pain After Total Knee Arthroplasty. Instr Course Lect. 2015;64:381-388. http://www.ncbi.nlm.nih.gov/pubmed/25745922. Accessed June 17, 2016

8. Westermann RW, Daniel JW, Callaghan JJ, Amendola A. Arthroscopic Management of Popliteal Tendon Dysfunction in Total Knee Arthroplasty. Arthrosc Tech. 2015;4(5):e565-e568. doi:10.1016/j.eats.2015.06.006.

9. de Simone V, Demey G, Magnussen RA, Lustig S, Servien E, Neyret P. Iatrogenic popliteus tendon injury during total knee arthroplasty results in decreased knee function two to three years postoperatively. Int Orthop. 2012;36(10):2061-2065. doi:10.1007/s00264-012-1631-5.

10. Kesman TJ, Kaufman KR, Trousdale RT. Popliteus tendon resection during total knee arthroplasty: an observational report. Clin Orthop Relat Res. 2011;469(1):7681. doi:10.1007/s11999-010-1525-z. 